\title{
Liraglutide alleviates diabetic cardiomyopathy by blocking CHOP-triggered apoptosis via the inhibition of the IRE- $\alpha$ pathway
}

\author{
YUQIANG JI $^{1}$, ZHAO ZHAO ${ }^{1}$, TIANZHI CAI ${ }^{2}$, PENGKANG YANG ${ }^{1}$ and MANLI CHENG ${ }^{1}$ \\ ${ }^{1}$ Department of Cardiovascular Medicine, First Hospital of Xi'an, Xi'an, Shaanxi 710002; \\ ${ }^{2}$ Department of Cardiovascular Medicine, The Affiliated Hospital of Xi'an Medical University, Xi'an, \\ Shaanxi 710077, P.R. China
}

Received July 6, 2013; Accepted February 6, 2014

DOI: $10.3892 / \mathrm{mmr} .2014 .1956$

\begin{abstract}
Clinically, diabetes mellitus is closely associated with and induces certain cardiovascular diseases. The aim of this study was to investigate endoplasmic reticulum (ER) stress-associated apoptosis of diabetic cardiomyopathy (DCM), and explore the protective mechanism of liraglutide. The DCM model was established with a high-fat diet and streptozotocin (STZ). Cardiac function was detected by echocardiogram examination and hematoxylin-eosin staining. ER stress unfolded protein response (UPR) hallmarks [inositol-requiring enzyme- $\alpha$ (IRE- $\alpha$ ), p-Perk and ATF6] and transcription factors were detected with western blotting. Apoptosis inducers CHOP, c-Jun amino terminal kinase (JNK) and casapse-12 were also examined with western blotting. The results indicated that liraglutide is capable of improving cardiac function in DCM rats $(\mathrm{P}<0.05)$. IRE- $\alpha$ expression was significantly increased in the DCM group compared with the control group $(\mathrm{P}<0.05)$, and liraglutide is capable of decreasing IRE- $\alpha$ expression. X-box transcription factor-1 (XBP-1) was significantly spliced in the model group, and downregulated in the liraglutide-treated group. CHOP protein was upregulated in the DCM group, but inactivated by liraglutide treatment. In conclusion, liraglutide is capable of protecting DCM and blocking CHOP-mediated ER stress by inhibiting the IRE- $\alpha$ UPR pathway.
\end{abstract}

\section{Introduction}

Diabetes mellitus is a serious and complex metabolic disease that affects the health of individuals worldwide (1). Numerous studies have demonstrated that diabetes mellitus is associated with, and induces, certain cardiovascular diseases (2).

Correspondence to: Professor Manli Cheng, Department of Cardiovascular Medicine, First Hospital of Xi'an, Fen Road 30, South Street, Xi'an, Shaanxi 710002, P.R. China

E-mail: chengmanlixa@yeah.net

Key words: liraglutide, diabetic cardiomyopathy, endoplasmic reticulum stress, apoptosis, cardiac function
Clinically, long-term diabetes may induce specific cardiomyopathy, which is known as diabetic cardiomyopathy (DCM) (3). Features of DCM include diastolic dysfunction and structural changes (4). To date, no specific drugs have been developed for the prevention or successful treatment of DCM. Furthermore, the mechanism underlying the pathogenesis of DCM remains elusive and treatment with specific drugs requires urgent investigation. Numerous factors may be involved in the pathogenesis of DCM, including cellular metabolism, defective calcium homeostasis, oxidative stress and others $(5,6)$.

Inside the cell, numerous stimuli including ischemia, hypoxia, abnormal protein synthesis and gene mutation may induce the pathological accumulation of unfolded proteins in the endoplasmic reticulum (ER), a condition referred to as ER stress (7). Certain complex homeostatic signaling pathways, including the unfolded protein response (UPR), have evolved to deal with ER stress (8). Certain studies have been reported that ER stress participates in diabetes-associated diseases, including diabetes mellitus, diabetic kidney disease and renal injury $(9,10)$.

Clinically, glucagon-like peptide-1 (GLP-1) has been identified as a therapeutic drug for the treatment of type 2 diabetes. However, the half-life of GLP-1 is particularly short, its analog, liraglutide, was identified in 2010 (11). Studies have indicated that liraglutide is beneficial for the improvement of cardiovascular function $(12,13)$. Therefore, in this study the correlation between ER stress and DCM, in addition to the role of liraglutide for ER stress and cardiac function in DCM were analyzed.

\section{Materials and methods}

Animals. In total, 60 male Wistar rats (weight, $200 \pm 25 \mathrm{~g}$ ) were purchased from the Animal Center of Xi'an Medical University (Xi'an, China). The rats were housed in plastic cages at room temperature $\left(20-22^{\circ} \mathrm{C}\right)$ and a relative humidity of $50-60 \%$ with a $12 \mathrm{~h}$ light/dark cycle. For eight weeks, 40 rats were fed a high-fat diet subsequent to treatment with two intraperitoneal injections of streptozotocin (STZ) for two weeks, once a week (30 mg/kg, Sigma-Aldrich, St. Louis, MO, USA). The other 20 rats were fed regular chow and injected with the same dosage of citrate buffer. In total 32 rats achieved the DCM standard of a stable fasting blood glucose 
(FBG) level $>7.8 \mathrm{mmol} / \mathrm{l}$. The other 20 rats with a normal diet all had FBG levels $<7.8 \mathrm{mmol} / \mathrm{l}$. Therefore, 52 rats survived five weeks after the initial injection. All remaining rats were used in the experiments. The study was approved by the Ethics Committee of the First Hospital of Xi'an, China.

Grouping. The remaining rats were divided into three groups: The non-DCM group (control, $n=10$ ), DCM rats without liraglutide treatment (model, $\mathrm{n}=14$ ), DCM rats with liraglutide $100 \mu \mathrm{g} / \mathrm{kg}$ treatment (LIRA, $\mathrm{n}=28$ ). FBG levels, body weight and cardiac function were measured at the baseline and throughout the eight weeks of treatment. All rats were anesthetized with chloral hydrate and sacrificed following eight weeks of treatment and heart tissue was obtained for the following experiments.

Echocardiogram examination. All 52 rats underwent an echocardiogram examination in order to verify the DCM. Each rat was anesthetized with ketamine $\mathrm{HCl}(50 \mathrm{mg} / \mathrm{kg}$; Tiangen, Beijing, China) and xylazine (10 mg/kg; Tiangen) prior to measurement. The echocardiogram (Sigma-Aldrich) examination was performed according to a method described previously (6). For each measurement, data from a minimum of three consecutive cardiac cycles were averaged. FBG levels were measured in spectrophotometry-based assays using commercially available kits (Sigma-Aldrich).

Hematoxylin-eosin (H\&E) staining. The left ventricle was isolated and sectioned into four slices along a plane parallel to the atrioventricular ring. The middle section was fixed in $4 \%$ buffered formalin, and paraffin-embedded sections $(4 \mu \mathrm{m})$ were prepared for $H \& E$ staining. The remaining section of the sample was stored at $-80^{\circ} \mathrm{C}$ prior to use in the western blotting.

Western blotting. Isolated cardiac tissues were lysed and separated with $15 \%$ SDS-PAGE. Western blotting was performed according to the study by Wang et al (14). The transferred proteins were incubated with 1:1,000 goat pAb anti-human CHOP, 1:2,000 mice mAb anti-human p-Perk, $1: 3,000$ mice $\mathrm{mAb}$ anti-human inositol-requiring enzyme- $\alpha$ (IRE- $\alpha$ ), 1:4,000 mice anti-Grp78, 1:2,000 mAb anti-human $\beta$-actin, 1:2,000 polyclonal $\mathrm{Ab}$ anti-human full-length and cleaved caspase-3 (Santa Cruz Biotechnology, Inc., Santa Cruz, CA, USA), 1:1,000 mAb anti-full length and spliced X-box transcription factor-1 (XBP1) (Enzo Life Sciences, Farmingdale, NY, USA), 1:1,000 mAb anti-full length and cleaved ATF6 (Santa Cruz Biotechnology, Inc.), 1:1,000 anti-eukaryotic translation initiation factor- $2 \alpha$ (eIF-2 $\alpha$ ) (Santa Cruz Biotechnology, Inc.) for $2 \mathrm{~h}$ at room temperature and subsequently incubated with the horseradish peroxidase (HRP)-conjugated secondary antibodies (Santa Cruz Biotechnology, Inc.). Reactive signals were visualized using an enhanced chemiluminescence kit (Pfizer, New York, NY, USA).

Statistic analysis. Quantitative analysis of immunoblot images was performed using computer-assisted software Image Total Tech (Pharmacia, Aachen, Germany). The image of the immunoblot was scanned using the Typhoon imager (Pharmacia), digitalized and saved as a TIF format.
The values of each target blot were evaluated. All data are presented as the mean \pm standard deviation. The individual groups were tested for differences using one-way analysis of variance repeated measurements, followed by independent samples t-test. $\mathrm{P}<0.05$ was considered to indicate a statistically significant difference.

\section{Results}

DCM rat model. To confirm whether the DCM model had been successfully established, cardiac function, FBG levels and model stability were assessed. For the DCM rats, the FBG level was significantly increased compared with the control group $(\mathrm{P}<0.05$; Fig. 1A). The fractional shortening (FS), and ejection fraction (EF) were significantly decreased (Fig. 1B and C), while the left ventricular end-diastolic diameter (LVEDD) and thickness of the left ventricular posterior wall (LVPW) were significantly increased (Fig. 1D and E), compared with the control group. A significant reduction in the E-wave velocity, significant increase in the A-wave velocity, and a significant decrease in the E/A ratio was observed (Fig. 1F). H\&E staining results indicated that diabetic cardiac tissues were disordered and a number of them were damaged (Fig. 2A and B). FGB levels and cardiac tissue H\&E staining results illustrated that the DCM model was established successfully.

Liraglutide alleviates cardiac damage in DCM rats. The results indicated that there was no significant changes for the FGB level in the LIRA group compared with the model group (Fig. 1A) (P>0.05). EF and FS were significantly enhanced by incubating with liraglutide (both $\mathrm{P}<0.05$ ) (Fig. 1B and C). Liraglutide was capable of decreasing LVPW and LVEDD levels significantly compared with the DCM rats (Fig. 1D and E) $(\mathrm{P}<0.05)$, and even achieved the same levels as the control group. Liraglutide also promoted the E/A ratio of the DCM rats (Fig. $1 \mathrm{~F})(\mathrm{P}<0.01)$. H\&E staining results also indicated that the disordered diabetic cardiac muscle fibers were repaired by treatment with liraglutide (Fig. 2).

Liraglutide alleviates IRE- $\alpha$-mediated ER stress. In order to investigate the specific mechanism for cardiac myocyte damage, the ER stress-associated protein Grp78 and UPR factors (including p-Perk, IRE-1 and ATF6) were detected by the western blotting. As shown in Fig. 3A, the Grp78 protein increased significantly compared with the control group, and the decreased to the normal level when treated with liraglutide (Fig. 3). In the model group the IRE- $\alpha$ factor was activated, but not triggered in the control group. Notably, IRE- $\alpha$ activity was inhibited when the rats were treated with liraglutide, and had a significantly lower level of expression when compared with the model group $(\mathrm{P}<0.05$, Fig. 3$)$. However, the p-Perk and ATF6 UPR levels did not alter significantly in any of the three groups $(\mathrm{P}>0.05)$.

Downstream ER stress-associated proteins of the UPR pathway, including spliced XBP1, tumor necrosis facto-associated receptor 2 (TRAF2) and eIF- $2 \alpha$ proteins, were also detected. In the model group, the levels of spliced XBP1 were significantly increased compared with the control group $(\mathrm{P}<0.05$, Fig. 4). When the rats were treated with liraglutide, the 
A
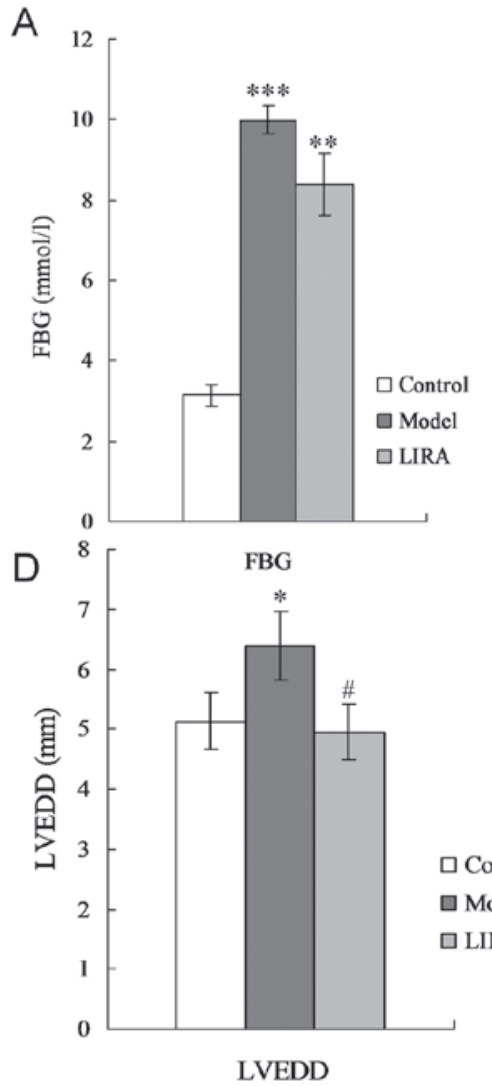

B

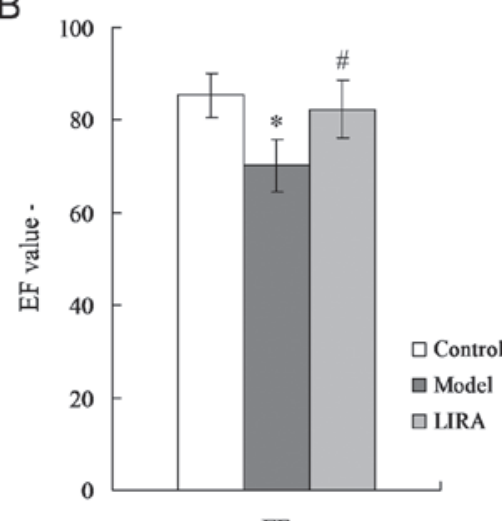

E
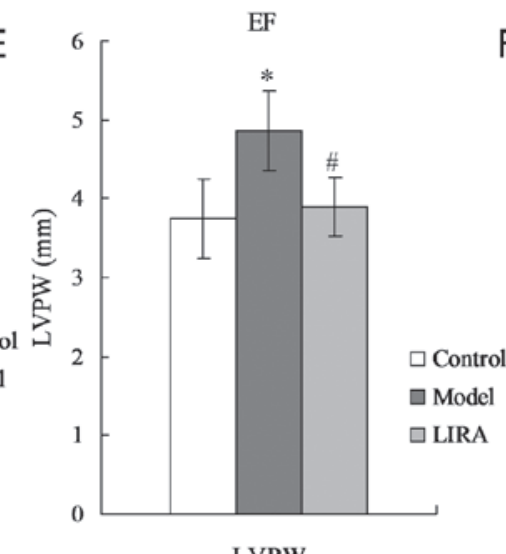

C

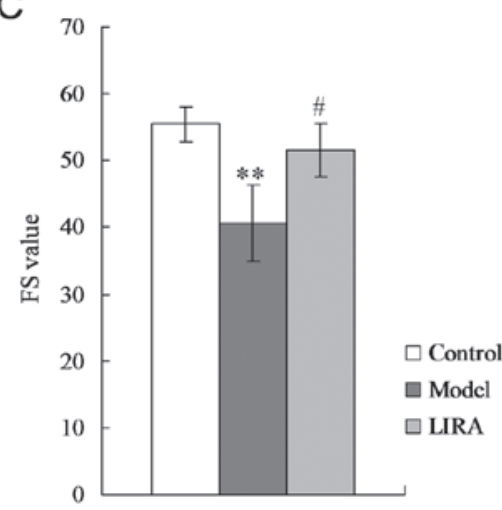

F 3 FS

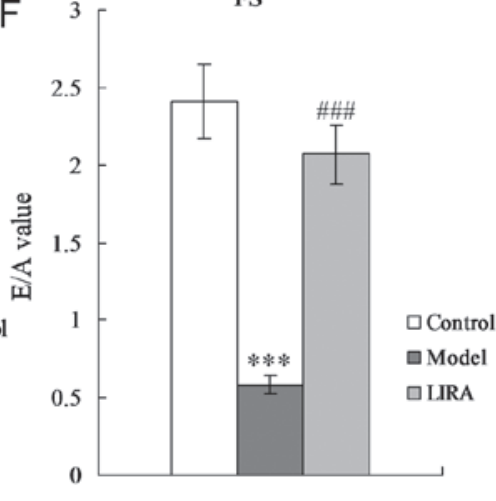

E/A

Figure 1. Results of echocardiogram and FBG. (A) FBG detection. (B) Echocardiographic analysis of EF. (C) Echocardiographic analysis of FS. (D) Echocardiographic analysis of LVEDD. (E) Echocardiographic analysis of LVPW. (F) E/A ratio. LVEDD, left ventricular end-diastolic diameter; LVPW, left ventricular posterior wall; FS, fractional shortening; EF, ejection fraction; FBG, fasting blood glucose; E/A, the ratio of E and A (E, peak early transmitral filling velocity during early diastole; A, peak transmitral atrial filling velocity during late diastole); ${ }^{*} \mathrm{P}<0.05,{ }^{* * *} \mathrm{P}<0.01$ and ${ }^{* * * *} \mathrm{P}<0.001$ vs. normal group rats; ${ }^{\#} \mathrm{P}<0.05,{ }^{\# \#} \mathrm{P}<0.01$ and ${ }^{\# \# \#} \mathrm{P}<0.001$ vs. model group rats.
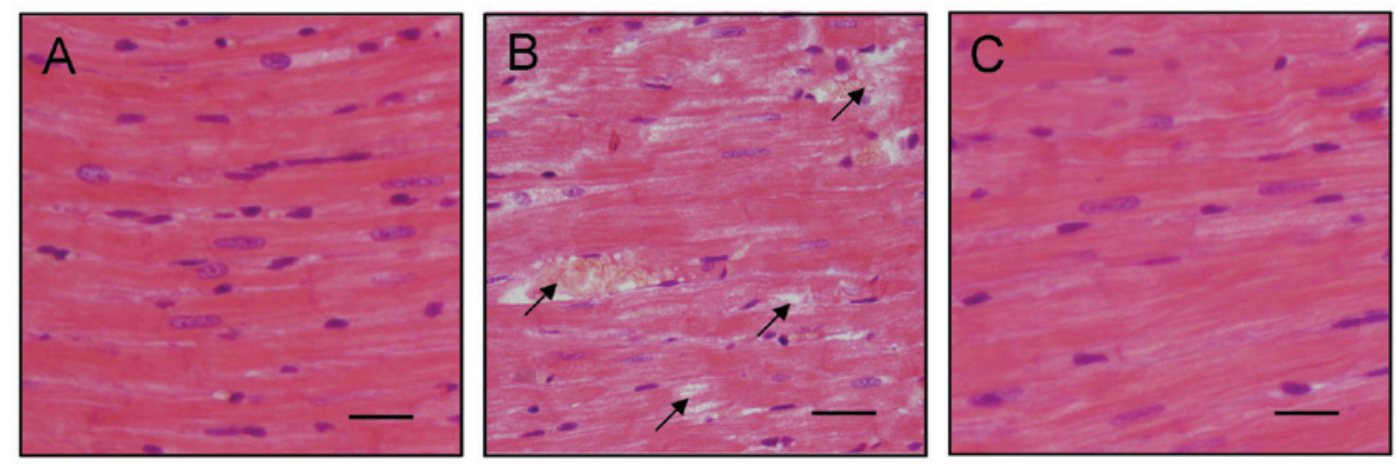

Figure 2. Histopathological examination in diabetic myocardium of the three groups. The normal and diabetic myocardium were stained with hematoxylin and eosin. (A) Myocardial tissue of normal rats without histopathological changes. (B) Myocardial sample of rats with DCM. (C) Liraglutide treated DCM rats. Arrows indicate regions with ischemic myocyte degeneration in the subendocardial, subepicardial region and papillary muscles of the myocardium. Scale bar, $50 \mu \mathrm{m}$. DCM, diabetic cardiomyopathy.

XBP1 levels decreased significantly compared with the model group, and returned to levels of the normal group (Fig. 4).

Liraglutide inhibits cardiac myocyte apoptosis by inhibiting CHOP expression. In order to confirm the key factor that induced ER stress-associated apoptosis of cardiac myocytes in the model group, the cellular levels of cleaved caspase-12, CHOP protein and phospho-c-Jun amino terminal kinase (p-JNK) were evaluated by individual western blotting (Fig. 4). CHOP protein levels were significantly increased in the model group compared with the control group $(\mathrm{P}<0.05$, Fig. 4). For the LIRA group, when treated with liraglutide, the CHOP protein levels were significantly decreased $(\mathrm{P}<0.05)$. No significant differences were identified among the three groups for the cleaved caspase- 12 and p-JNK proteins $(\mathrm{P}>0.05)$. These results indicated that $\mathrm{CHOP}$ was activated in the DCM rat model, and triggered apoptosis. Treatment with liraglutide blocked the CHOP-triggered apoptosis via IRE- $\alpha$ UPR in the DCM rat model. 
A
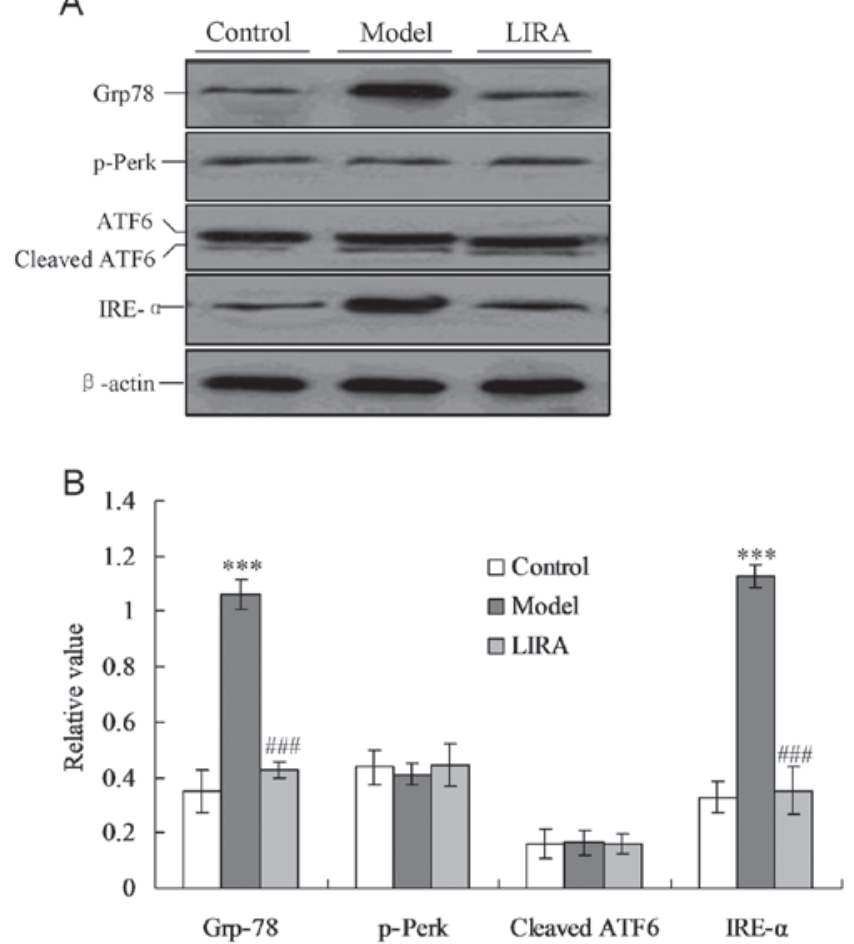

C
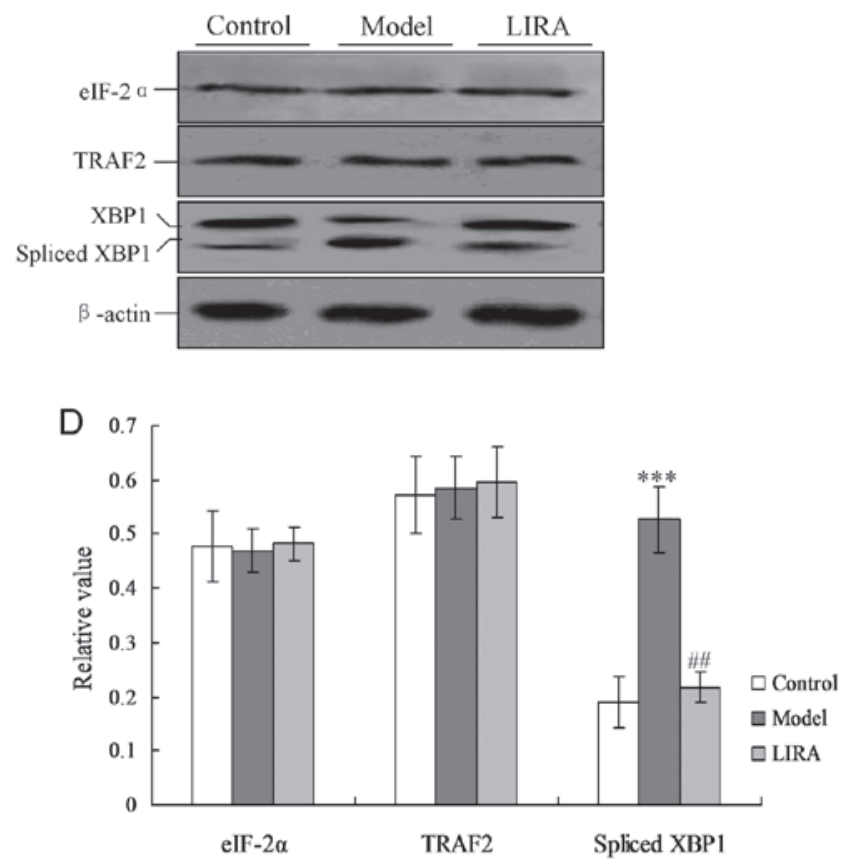

Figure 3. Detection of ER-associated UPR pathway proteins and downstream transcription factors. (A) Detection of UPR proteins, including IRE- $\alpha$, p-Perk and cleaved ATF6. (B) Statistical analysis of UPR proteins analyzed by western blotting. (C) Detection of UPR downstream transcription factors, including spliced XBP1, TRAF2, and eIF-2 $\alpha$ proteins. (D) Statistical analysis of UPR associated factors analyzed by western blotting. The average gray value of each preparation was calculated by the gray numerical value of each blot versus that of $\beta$-actin. The average data of each preparation were evaluated from three independent blots and represented as the mean \pm stadnard deviation. ${ }^{*} \mathrm{P}<0.05,{ }^{* * *} \mathrm{P}<0.01$ and ${ }^{* * *} \mathrm{P}<0.001$ vs. normal group rats; ${ }^{*} \mathrm{P}<0.05$, ${ }^{\# \#} \mathrm{P}<0.01$ and ${ }^{\# \# \#} \mathrm{P}<0.001$ vs. model group rats. ER, endoplasmic reticulum; UPR, unfolded protein response, eIF-2 $\alpha$, eukaryotic translation initiation factor-2 $\alpha$; XBP1, X-box transcription factor-1; TRAF2, tumor necrosis factor receptor-associated factor 2; model, diabetic cardiomyopathy (DCM) rats without liraglutide treatment; LIRA, DCM rats treated with $100 \mu \mathrm{g} / \mathrm{kg}$ liraglutide; IRE- $\alpha$, inositol-requiring enzyme- $\alpha$.
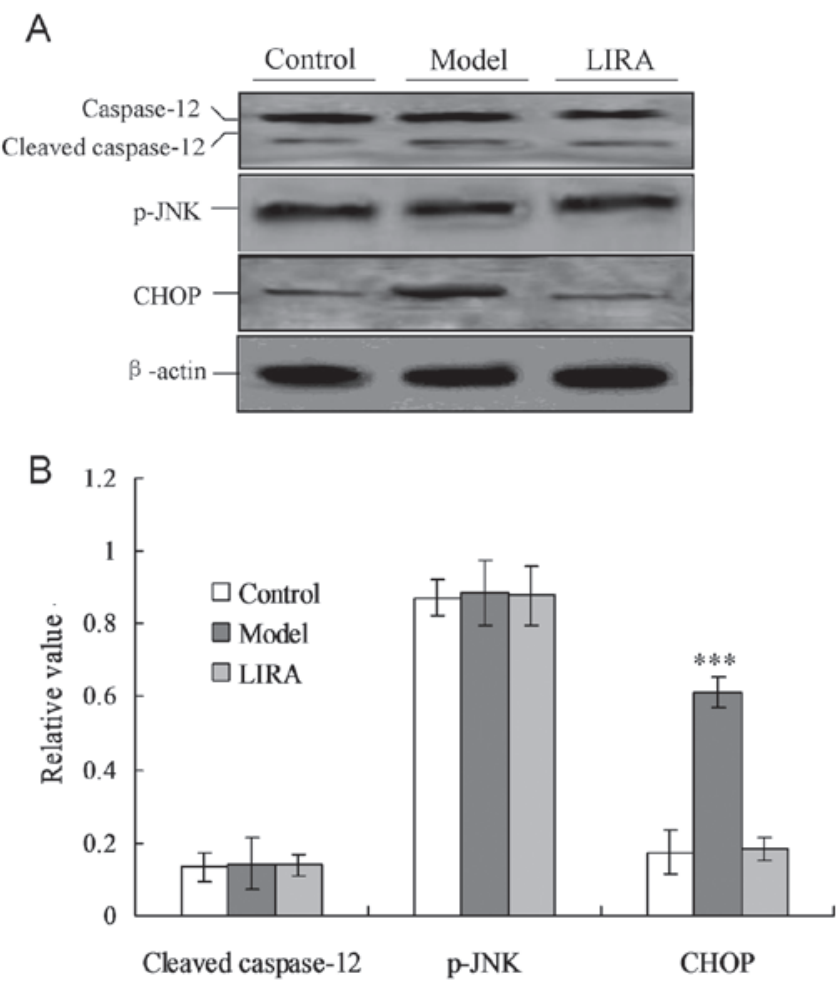

Figure 4. ER-stress associated pro-apoptotic factor detection. (A) The levels of p-JNK, cleaved caspase-12 and CHOP protein in three groups were evaluated by western blotting. (B) Statistical analysis of the western blotting assay. The average gray value of each preparation was calculated by the gray numerical value of each blot versus that of $\beta$-actin. The average data of each preparation were evaluated from three independent blots and represented as the mean \pm standard deviation. ${ }^{*} \mathrm{P}<0.05,{ }^{* *} \mathrm{P}<0.01$ and ${ }^{* * *} \mathrm{P}<0.001$ vs. normal group rats; ${ }^{\#} \mathrm{P}<0.05,{ }^{\# \#} \mathrm{P}<0.01$ and ${ }^{\# \# \#} \mathrm{P}<0.001$ vs. model group rats. ER, endoplasmic retculum; p-JNK, phospho-c-Jun amino terminal kinase; model, diabetic cardiomyopathy (DCM) rats without liraglutide treatment; LIRA, DCM rats treated with $100 \mu \mathrm{g} / \mathrm{kg}$ liraglutide.

\section{Discussion}

Numerous studies have demonstrated that there is correlation a between diabetes and cardiomyopathy (15-17). Epidemiological investigations have also suggested that diabetes mellitus increases the incidence of cardiac dysfunction and heart failure. DCM is characterized by diastolic and systolic dysfunction, and hyperglycemia (18). DCM has been considered to be one of the most important causes of cardiac dysfunction and heart failure in the progress of diabetes mellitus (19). In the present study, a DCM model was successfully established, and the FBG levels and cardiac myocyte functions were detected. Induction by STZ to form the DCM model is the most widely used method for establishing a diabetes model. The combination of STZ and a high-fat diet is particularly suitable for the examination of the pathophysiology of DCM $(20,21)$. The method used in the present study to establish the DCM model is consistent with that used in previous studies (22). In the present study, the effects of liraglutide on cardiac damage and myocardial apoptosis in DCM rats were investigated. Additionally, the potential mechanism involved in this process was also discussed.

Studies have indicated that hyperglycemia-induced ER stress is important role in the DCM (23). In this study, the levels of Grp78 protein were initially detected in the DCM rats, 
which may represent the appearance of ER stress. The results indicated that the Grp78 level was higher in the model group, thus, the ER stress-associated UPR pathway proteins, including p-Perk, ATF6 and IRE- $\alpha$, were detected. Notably, the IRE- $\alpha$ level was also significantly enhanced compared with the normal group, but no changes were observed for the p-Perk and ATF6 proteins in the three groups. The activation of Perk phosphorylated eIF-2 $\alpha$ suppresses protein synthesis (24). Activation of the RNase activity of IRE- $\alpha$ initiates the splicing of XBP-1 into spliced variant XBP-1 mRNA, which is subsequently translated into a potent transcription factor (25). A combination of ATF6 and the spliced variant of XBP1 positively regulate a variety of UPR target gene expression, including several ER resident chaperones $(24,25)$. Therefore, the downstream factors, including spliced XBP-1, TRAF2 and eIF-2 $\alpha$, were examined using western blotting. The results indicated that only the XBP-1 factor was highly spliced in the model group compared with the normal group. Liraglutide has been used extensively to treat hypertension, heart failure and other cardiovascular diseases, which not only improves cardiac function, but also resists apoptosis. Thus DCM rats were treated with liraglutide in order to observe its effects on ER stress and cardiac function. The results demonstrated that the addition of liraglutide may improve cardiac function markedly, and significantly inhibit XBP-1 splicing. Therefore, it was hypothesized that liraglutide is important in inhibiting IRE- $\alpha$ and XBP-1-mediated ER stress.

Three apoptotic pathways have been thoroughly investigated in association with ER stress (26). The most significant ER stress-induced apoptotic pathway is mediated through CHOP/GADD153, a transcription factor induced by XBP-1, ATF4, and ATF6. Another pathway is the JNK pathway, which is mediated by TRAF2. TRAF2 may interact with IRE- $\alpha$ and apoptosis signal-regulating kinase-1 (ASK1), which subsequently phosphorylates and activates JNK. Caspase-12 mediated cell apoptosis was also investigated, which is only sensitive to ER stress-induced apoptosis. Therefore, the three apoptotic pathways were investigated and it was observed that only CHOP participated in ER stress-associated apoptosis. Furthermore, liraglutide blocked any increase in the level of CHOP protein in addition to inhibiting apoptosis in the DCM model. Notably, though liraglutide is capable of resisting ER stress, it is not capable of downregulating high glucose levels in DCM rats.

In conclusion, the present study confirms that DCM is an important stimulus for the ER stress response of the myocardium cells. It was observed that liraglutide is capable of blocking CHOP-mediated ER stress by inhibiting the IRE- $\alpha$ UPR pathway. This may provide the novel therapeutic strategies or methods for the clinical DCM.

\section{Acknowledgements}

This study was supported by the Science and Technology Research and Development Program of Shaanxi Province of China (grant no. 2011K14-08-04).

\section{References}

1. Zhang SY, Zhang QJ, Zhang LH, Li CG and Jiang HQ: Expression of ghrelin and leptin during the development of type 2 diabetes mellitus in a rat model. Mol Med Rep 7: 223-228, 2013.
2. Yamagishi S: Cardiovascular disease in recent onset diabetes mellitus. J Cardiol 57: 257-262, 2010.

3. Diao XH, Shen E, Wang XX and Hu B: Differentially expressed microRNAs and their target genes in the heart of streptozotocin-induced diabetic mice. Mol Med Rep 4: 633-640, 2011.

4. Dhalla NS, Rangi S, Zieroth S and Xu YJ: Alterations in sarcoplasmic reticulum and mitochondrial functions in diabetic cardiomyopathy. Exp Clin Cardiol 17: 115-120, 2012.

5. Boudina S and Abel ED: Diabetic cardiomyopathy revisited. Circulation 115: 3213-3223, 2007.

6. Nartprayut K, U-Pratye Y, Kheolamai P, Manochantr S, Chayosumrit M, Issaragrisil S and Supokawej A: Cardiomyocyte differentiation of perinatally-derived mesenchymal stem cells. Mol Med Rep 7: 1465-1469, 2013.

7. Kim I, Xu W and Reed JC: Cell death and endoplasmic reticulum stress: disease relevance and therapeutic opportunities. Nat Rev Drug Discov 7: 1013-1030, 2008.

8. Marciniak SJ and Ron D: Endoplasmic reticulum stress signaling in disease. Physiol Rev 86: 1133-1149, 2006.

9. Liu G, Sun Y, Li Z, Song T, Wang H,Zhang Y and Ge Z: Apoptosis induced by endoplasmic reticulum stress involved in diabetic kidney disease. Biochem Biophys Res Com 370: 651-656, 2008.

10. He DQ, Li JQ, Zhao JY, Fei J and Zhang XM: C/EBP homologous protein induces mesangial cell apoptosis under hyperglycemia. Mol Med Rep 7: 445-448, 2013.

11. Drucker DJ, Dritselis A and Kirkpatrick P: Liraglutide. Nat Rev Drug Discov 9: 267-268, 2010.

12. Ban K, Noyan-Ashraf MH, Hoefer J, Bolz SS, Drucker DJ and Husain M: Cardioprotective and vasodilatory actions of glucagon-like peptide 1 receptor are mediated through both glucagon-like peptide 1 receptor-dependent and independent pathways. Circulation 117: 2340-2350, 2008.

13. Schisano B, Harte AL, Lois K, et al: GLP-1 analogue, liraglutide protects human umbilical vein endothelial cells against high glucose induced endoplasmic reticulum stress. Regul Pept 174: 46-52, 2012.

14. Wang X, Dong CF, Shi Q, et al: Cytosolic prion protein induces apoptosis in human neuronal cell SH-SY5Y via mitochondrial disruption pathway. BMB Rep 42: 444-449, 2009.

15. Movahed MR, Hashemzadeh M and Jamal MM: Diabetes mellitus is a strong, independent risk for atrial fibrillation and flutter in addition to other cardiovascular disease. Int $\mathrm{J}$ Cardiol 105: 315-318, 2005.

16. Wu FH, Jin ZG and Jin J: Hypoglycemic effects of glabridin, a polyphenolic flavonoid from licorice, in an animal model of diabetes mellitus. Mol Med Rep 7: 1278-1282, 2013.

17. Aziz MT, EI Ibrashy IN, Mikhailidis DP, et al: Signaling mechanisms of a water soluble curcumin derivative in experimental type 1 diabetes with cardiomyopathy. Diabetol Metab Syndr 5: $13,2013$.

18. Galderisi M: Diastolic dysfunction diabetic cardiomyopathy: evaluation by Doppler echocardiography. J Am Coll Cardiol 48: 1548-1551, 2006

19. Wu TT, Dong Z, Geng J, et al: Valsartan protects against ER stress-induced myocardial apoptosis via CHOP/Puma signaling pathway in streptozotocin-induced diabetic rats. Eur J Pharm Sci 42: 496-502, 2011.

20. Lin G, Craig GP, Zhang L, et al: Acute inhibition of Rho-kinase improves cardiac contractile function in streptozotocin-diabetic rats. Cardiovasc Res 75: 51-58, 2007.

21. Yang HY, Fan SR, Song DP, et al: Long-term streptozotocin-induced diabetes in rats leads to severe damage of brain blood vessels and neurons via enhanced oxidative stress. Mol Med Rep 7: 431-440, 2013.

22. Zhang M, Lv XY, Li J, Xu ZG and Chen L: The characterization of high-fat diet and multiple low-dose streptozotocin induced type 2 diabetes rat model. Exp Diabetes Res 2008: 704045, 2008.

23. Mulhern ML, Madson CJ, Danford A, Ikesugi K, Kador PF and Shinohara T: The unfolded protein response in lens epithelial cells from galactosemic rat lenses. Invest Opthalmol Vis Sci 47: 3951-3959, 2006.

24. Moenner M, Pluquet O, Bouchecareilh M and Chevet E: Integrated endoplasmic reticulum stress response in cancer. Cancer Res 67: 10631-10634, 2007.

25. Pillai S: Birth pangs: the stressful origins of lymphocytes. J Clin Invest 115: 224-227, 2005.

26. Li MC, Liu Z, Zhuan L, Wang T, Guo SM, Wang SG, Liu JH and Ye ZQ: Effects of apocynin on oxidative stress and expression of apoptosis-related genes in testes of diabetic rats. Mol Med Rep 7: 47-52, 2013. 